\title{
The negative impact of market integration on cardiovascular fitness, inflammation markers and metabolic variables in Shuar Women living in Ecuadorian Amazonian Rainforest
}

\author{
Folke Lindgärde ${ }^{1 *}$ and Kerstin Johnsson ${ }^{2}$ \\ ${ }^{1}$ The Department of Vascular Medicine, Malmo University Hospital, Lund University, 21428 Malmo, Sweden \\ ${ }^{2}$ Hospital Vozandes del Oriente Shell, Shell Mera, Ecuador, Sweden
}

\begin{abstract}
Purpose: Physical fitness is inversely connected with risk markers associated with cardiovascular diseases. Whether individuals in remote Shuar villages in the Amazonian rainforest exhibit a decline in fitness related to market integration has not been clarified.

Methods: 33 healthy Shuar women were prospectively followed for seven years and were compared with 22 women, who had migrated from the rainforest to an urban area (Shell community). A modified Harvard step test was used to evaluate the cardio-respiratory fitness.

Results: Anthropomorphic variables of the 33 subjects were unchanged at follow-up examination. These women had in comparison to women who had moved to the Shell community lower body fat / body mass index ratio, lower diastolic blood pressure and serum insulin level.

After three minutes exercise heart rate had increased more in the Shell group and correlated positively with the numbers of years since the women left the rainforest. Body fat, systolic and diastolic blood pressure, serum insulin and high-sensitive CRP correlated significantly with heart rate.

Conclusion: The outcome of transition in life style, from collecting wild food products and performing manual agriculture, to an urbanized style is characterized by a deterioration of physical fitness.
\end{abstract}

\section{Introduction}

Lifestyle in isolated Amerindian populations in the Amazonian region seems to protect against the development of hypertension, obesity and diabetes [1], while high rates of cardiovascular risk factors are found when an Indian tribe in the Amazonian region has suffered cultural changes [2]. In remote villages in the rainforest, the first step of transition in lifestyle from hunter-gatherer societies to an agricultural lifestyle is associated with increased body fat mass with concomitant increase in circulating insulin and leptin [3].

The Shuar are an indigenous population concentrated in the southeastern Amazonian rain forest of Ecuador depending on subsistence activities, particularly horticultural production.

The present study was performed in women living in five Shuar villages, located at an altitude of $700 \mathrm{~m}$ approximately 160 kilometers southeast from Shell Mera in the Morona Santiago region in Ecuador.

The goals of this study are as follows: 1) to investigate potential differences in anthropomorphic and metabolic variables in Shuar women, obtained at a survey in 2003 [3] and at a follow-up study seven years later; and 2) to evaluate whether physical fitness, measured as heart rate at a step test procedure, and indicators of cardiovascular disease are influenced by market integration, i.e., migration from remote Shuar villages to the Shell Mera community, a small town in close proximity to the rainforest.

\section{Materials and methods}

\section{Subjects}

The study population consisted of two groups. The first group consisted of 59 women living in five Shuar communities in the Ecuadorian Amazonian rainforest that were examined in 2003 [3]. A follow-up study was performed during one day in the largest of the five villages, in the Yuwientsa community, in 2010. Among the study population, one women had died, one women was in the hospital, nine women had moved to other communities, and eight women from the four small villages did not show up, two women were pregnant, three women were unable to perform the step test and two women were unwilling to take part in the second investigation. A total of 33 subjects (age range 27-66, with a mean age of 43 years) consented to participate in the second study. These 33 women did not differ significantly from the remaining subjects who participated in the baseline study regarding measurements of body weight, body mass index, waist circumference and body fat mass. None of the women were pregnant or lactating.

Correspondence to: Folke Lindgärde, The Department of Vascular Medicine, Malmo University Hospital, Lund University, 21428 Malmo, Sweden, Tel: +46761055444; E-mail: folke.lindgarde@insatnet.nu

Received: April 01, 2017; Accepted: April 18, 2017; Published: April 21, 2017 
The second study group consisted of 22 Shuar women of the same age (range 26-66, with a mean of 39 years) who were born and lived in the rainforest (range 9-55, with a mean of 22 years) and later had settled in an urban area, in the Shell Mera community, and had lived there for at least 4 years (range $4-45$, with a mean of 17 years). The examinations of these women took place at the hospital at Shell during the same month, as the follow-up study was performed in the rainforest.

Approval for the original study was given by Hospital Vozandes, in Quito, Ecuador the follow-up study was approved separately by Minesterio Provincial de Salude de Morona Santiago.

\section{Clinical parameters}

The examination of all women was conducted in the morning after an overnight fast. The examination procedures were the same in 2003 and 2010. Height was measured without shoes. Body mass index $\left(\mathrm{BMI} ; \mathrm{kg} / \mathrm{m}^{2}\right.$ ) was calculated according to the standard formula. Waist circumference was measured at the level of the midpoint between the lower costal margin and the anterior superior iliac crests.

Body adiposity was measured by a bioelectrical impedance method (Biodynamic Model 310e; Biodynamic Research Inc., Seattle, WA). Measurements were performed with subjects lying on a couch for five minutes, and the electrodes were placed on the dorsal surfaces of the right hand and foot. Blood pressure was measured after placing a cuff over the upper right arm after a five-minutes rest.

\section{Biochemical parameters and procedure}

At the Yuwientsa community the blood was centrifuged, the serum was stored in an ice cooler with a blue pack ice at $4^{\circ} \mathrm{C}$ and was then transported by air to the Shell Mera hospital and stored at $-20^{\circ} \mathrm{C}$. Within a week, samples were brought to Sweden on dry ice by air and stored at $-80^{\circ} \mathrm{C}$ until further analyses. Blood glucose measurements were performed on whole fresh capillary blood using a Hemocue B-Glucose Analyzer (HemoCue AB, Ängelholm, Sweden). Serum insulin, haptoglobin, high-sensitivity C-reactive protein (hsCRP), orosomucoid, cholesterol, LDL and HDL-cholesterol and triacylglycerol were analyzed at the Department of Clinical Chemistry, University Hospital, Lund University, Sweden.

A modified Harvard step test was used to evaluate the cardiorespiratory fitness. With respect to the height of the women, approximately $150 \mathrm{~cm}$, a platform $30 \mathrm{~cm}$ high was used. The stepping rate was paced by a metronome at a stepping rate of 45 steps / min, and the duration was three minutes. Heart rate was measured before the test started, immediately after the exercise ended and after three minutes rest.

\section{Statistical analysis}

All results are presented as the mean values $\pm \mathrm{SD}$. Changes in values between 2003 and 2010 were comparing by using the Wilcoxon signed rank test. Differences between the groups at the survey in 2010 were analyzed by the Mann-Whitney $\mathrm{U}$ test. The correlation coefficients between two variables were determined by Spearman rank analysis. A p-value of less than 0.05 was considered significant. Multiple regression analysis was undertaken to examine which variables predicted physical fitness. The selected variables were forced into the model based on analysis (Table 1). All statistical analyses were conducted by using the statistical package STATVIEW (version 5.0.1, for Macintosh; SAS Institute Inc., Cary, NC).

\section{Results}

Comparisons of variables obtained at baseline and at followup examination seven years later in subjects from Shuar villages

There were no differences in height, body weight, waist circumstance, body mass index (BMI), lean body mass, fat mass and concentrations of total blood cholesterol and triacylglycerol. Blood pressure values were lower at the follow-up examination (Table 2).

\section{Descriptive characteristics of the study sample from the survey in 2010 stratified by rainforest and urban residences}

The women from the Shell community had higher body fat mass/ BMI ratio, diastolic blood pressure and mean values of serum insulin and orosomucoid concentrations compared to women from the rain forest. In contrast, women from the Shuar villages had higher concentrations of triacylglycerol. All other variables, including blood glucose levels, were not significantly different from each other (Table 3).

\section{Heart rate increase at the step test in women from the Shuar villages and participants from Shell community}

Resting heart rate was similar in the two groups. However, after three minutes of exercise, heart rate had increased significantly more in the Shell group: 33 vs. 20 beats / $\min (P=0.017)$. There were no correlations between age and heart rate increase in either of the two groups (Table 1).

\section{Heart rate at the step test and number of years as residents at the Shell Community}

Spearman rank correlation tests were used to analyze the associations between the number of years since the women left the rainforest and the heart rate measured at the step test. There was a positive significant correlation between the number of years since the participants migrated from the rainforest to the Shell community and

Table 1. Heart rate (HR) beats per minute $(M \pm S D)$ at a modified Harvard Step test. Exercise was performed during three minutes, 45 steps per minute. HR increase (heart rate after three minutes exercise - heart rate at start).

\begin{tabular}{|c|c|c|c|}
\hline & Traditional Shuar villages & Shell community & \\
\hline & $(\mathrm{n}=33)$ & $(\mathrm{n}=22)$ & $\mathrm{P}$ \\
\hline Resting HR & $68 \pm 7$ & $66 \pm 9$ & NS \\
\hline HR after exercise & $88 \pm 12$ & $99 \pm 9$ & 0.034 \\
\hline HR after 3 min rest & $71 \pm 9$ & $76 \pm 14$ & NS \\
\hline HR increase & $20 \pm 9$ & $33 \pm 13$ & 0.017 \\
\hline
\end{tabular}

Table 2. Anthropomorphic data, blood lipids and blood pressure values from the survey in 2003 and the results seven years later at the follow-up investigation in 33 Ecuadorian Amerindian women living in five villages in the Amazonian rainforest.

\begin{tabular}{|c|c|c|c|}
\hline & $\mathbf{2 0 0 3}$ & $\mathbf{2 0 1 0}$ & $\mathbf{P}$ \\
\hline Age $(\mathrm{yr})(\mathrm{range})$ & $34.1(20-60)$ & $43.2(27-67)$ & $\mathrm{NS}$ \\
\hline Weight $(\mathrm{kg})$ & $56.2 \pm 6.6$ & $57.4 \pm 8.3$ & $\mathrm{NS}$ \\
\hline Height $(\mathrm{cm})$ & $151 \pm 4$ & $150 \pm 5$ & $\mathrm{NS}$ \\
\hline BMI $\left(\mathrm{kg} / \mathrm{m}^{2}\right)$ & $24.3 \pm 2.3$ & $25.2 \pm 3.1$ & $\mathrm{NS}$ \\
\hline Waist circumference $(\mathrm{cm})$ & $84.0 \pm 8.6$ & $84.5 \pm 87.6$ & $\mathrm{NS}$ \\
\hline Fat mass $(\mathrm{kg})$ & $13.8 \pm 5.0$ & $14.1 \pm 4.0$ & $\mathrm{NS}$ \\
\hline Lean body weight $(\mathrm{kg})$ & $41.4 \pm 4.5$ & $42.8 \pm 5.6$ & $\mathrm{NS}$ \\
\hline Cholesterol $(\mathrm{mmol} / \mathrm{L})$ & $4.67 \pm 0.58$ & $4.86 \pm 0.82$ & $\mathrm{NS}$ \\
\hline Triacylglycerol $(\mathrm{mmol} / \mathrm{L})$ & $2.19 \pm 1.0$ & $1.83 \pm 0.64$ & $\mathrm{NS}$ \\
\hline Systolic blood pressure $(\mathrm{mm} \mathrm{Hg})$ & $111 \pm 13$ & $108 \pm 13$ & 0.003 \\
\hline Diastolic blood pressure $(\mathrm{mm} \mathrm{Hg})$ & $72 \pm 9.0$ & $67 \pm 8$ & 0.024 \\
\hline
\end{tabular}


Table 3. Characteristics $(M \pm S D)$ of women living in traditional Shuar villages $(n=33)$ and women who have moved from the rainforest to the Shell community $(n=22)$.

\begin{tabular}{|c|c|c|c|}
\hline Variable & Shuar villages & Shell community & P \\
\hline Age & $43.2 \pm 11.0$ & $38.8 \pm 8.0$ & NS \\
\hline Body weight & $57.4 \pm 8.3$ & $56.6 \pm 3.5$ & NS \\
\hline Waist circumference & $84.5 \pm 8.6$ & $81.1 \pm 9.1$ & NS \\
\hline Body mass index & $25.0 \pm 3.1$ & $24.5 \pm 3.5$ & NS \\
\hline Body fat kg & $14.1 \pm 4.0$ & $15.3 \pm 4.5$ & NS \\
\hline Body fat/BMI & $0.55 \pm 0.12$ & $0.62 \pm 0.14$ & 0.02 \\
\hline Systolic BP mm Hg & $108 \pm 13$ & $110 \pm 17$ & NS \\
\hline Diastolic BP mm Hg & $67 \pm 8$ & $72 \pm 9$ & 0.028 \\
\hline Blood glucose $\mathrm{mmol} / \mathrm{L}$ & $5.50 \pm 0.54$ & $5.56 \pm 0.52$ & NS \\
\hline Serum insulin mlE/L & $3.55 \pm 3.21$ & $6.15 \pm 5.30$ & 0.012 \\
\hline Haptoglobin $\mathrm{g} / \mathrm{L}$ & $1.21 \pm 0.30$ & $1.34 \pm 0.38$ & NS \\
\hline Orosomucoid g/L & $0.49 \pm 0.14$ & $0.63 \pm 0.23$ & 0.035 \\
\hline hs-CRP mg/L & $1.85 \pm 2.8$ & $2.82 \pm 3.75$ & NS \\
\hline Cholesterol mmol/L & $4.86 \pm 0.82$ & $4.76 \pm 0.43$ & NS \\
\hline HDL cholesterol mmol/L & $1.03 \pm 0.29$ & $1.12 \pm 0.40$ & NS \\
\hline LDL cholesterol mmol/L & $2.89 \pm 0.80$ & $3.03 \pm 1.12$ & NS \\
\hline Triacylglycerol mmol/L & $1.83 \pm 0.70$ & $1.43 \pm 1.00$ & 0.023 \\
\hline
\end{tabular}

Table 4. Multiple regression analysis in 55 Shuar women with the indicated variables obtained at the survey in 2010 as independent variables and heart rate after three minutes of exercise at a step test as the dependent variable.

\begin{tabular}{|c|c|c|c|c|}
\hline & Coefficient & SE & Standardcoefficient & P \\
\hline Intercept & 28.25 & 16.21 & 1.743 & 0.5505 \\
\hline BP systolic & 0.322 & 0.534 & 0.479 & 0.0014 \\
\hline Body fat kg. & 0.513 & 0.0014 & 0.097 & 0.5505 \\
\hline hs-CRP & 2.033 & 0.790 & 0.394 & 0.0148 \\
\hline Insulin & 0.299 & 0.436 & 0.098 & 0.497 \\
\hline
\end{tabular}

the heart rate after three minutes exercise $(\mathrm{Rho}=0.69 ; \mathrm{P}=0.0048)$ as well as after three minutes rest $(\mathrm{Rho}=0.627 ; \mathrm{P}=0.0097)$.

\section{Relationship between heart rate after three minutes of exercise and variables}

When evaluating participants from the two groups together significant associations were found between fitness (heart rate at three minutes of exercise) and body fat $(\mathrm{r}=0.317 ; \mathrm{P}=0.038)$, systolic blood pressure $(\mathrm{r}=0.371 ; \mathrm{P}=0.014)$, diastolic blood pressure $(\mathrm{r}=0.35 ; \mathrm{P}=$ $0.02)$, serum concentrations of insulin $(r=0.31 ; P=0.043)$ and hs-CRP $(\mathrm{r}=0.454 ; \mathrm{P}=0.005)$ obtained at the survey at 2010. There were no significant relations to age, blood glucose and serum concentrations of orosomucoid, haptogloin and lipids (Table 3).

\section{Predictors of the heart rate at the step test after three minutes of exercise}

Multiple regression analysis was performed with pulse rate after three minutes of exercise as the dependent variable and variables from both study groups together that were significantly associated with fitness as independent variables. Systolic blood pressure $(P=0.0014)$ and serum hs-CRP $(\mathrm{P}=0148)$ significantly predicted cardiovascular fitness (Table 4).

\section{Discussion}

Several important and novel findings have been made in this study of Shuar women living in the Amazonian rainforest that warrant discussion. First, seven years after the baseline examination body compartments and blood lipid concentrations are unaltered (Table 2). The most likely explanation to these findings is that individuals in subsistence populations engage in physically demanding work well into old-age, a lifestyle that might counterbalance the senescent decline in lean body mass and cardiovascular fitness observed in industrialized populations [4]. This hypothesis was strengthened by the observation that there was no age-related decline in physical fitness measured at the follow-up survey.

Second, those social and cultural changes that occur with market integration are in the present study associated with a decline in physical fitness that interacts with the numbers of years since the women left the villages in the rainforest and settled down as city-dwellers. Furthermore, these Shell Mera residents had higher body fat/body mass ratio and serum concentrations of insulin and orosomucoid. However, the elevation of serum insulin concentration was not associated with a concomitant derangement of blood glucose.

Elevated C-reactive protein (CRP) is a well-recognized clinical marker of inflammation that is associated with increased incidence of cardiovascular diseases [5]. The epidemiological data suggest that physical activity levels are inversely associated with markers of inflammation including CRP. LaMonte et, al. observed a negative association between CRP and fitness among Native American women [6]. Amount of fat mass is undeniably one of the strongest correlations of circulating inflammatory biomarkers. In the present study impairment of physical fitness was associated with amount of body fat, systolic blood pressure level and serum concentrations of insulin and CRP (Table 1).

When a multiple regression analysis included these four variables as independent predictors, systolic blood pressure and CRP were the only predictors of physical fitness (Table 4).

The discordant mean triacylglycerol concentrations, 1.77 (Shuar villages) and $1.43 \mathrm{mmol} / \mathrm{L}$ (Shell community) (Table 3), demonstrates the importance of diet. The mean value among Shell participants is identical with the triacylglycerol concentration, 1.46, in Amerindian women living in Lima, Peru [7]. A direct comparison between the food compositions in the two Shuar groups is hampered by the lack of detailed dietary data. However, a dietary analysis from the 70-ties estimated that $75 \%$ of their calories were derived from horticulture $17 \%$ from hunting and 5\% from fishing [8]. The fat intake is very low and probably not more than about $10 \%$ of the energy intake. This assumption is supported by data reported in a literature review of diet and nutritional status of Amerindians living in Amazonia [9]. The dietary intake of the women in Lima determined by 24 -hour recall in 1999 [6] indicated that approximately $20 \%$ of their calories were derived from fat, $12 \%$ from protein and $69 \%$ from carbohydrates, with a high intake of products containing refined carbohydrates and sugar. It is likely that the Shell women had adopted a diet similar to the Lima women.

This study was performed to provide data in an important area where data do not currently exist. To our knowledge this is the first prospective study of body compartments, blood pressure and blood lipids analyzed in a subsistence level population in the Amazonian region. In spite of the women being seven years older at the followup survey these parameters were unaltered other than lower blood pressure levels. Furthermore, they demonstrated higher levels of cardiovascular fitness than Shuar women of the same age who were urban dwellers. Low physical activity has been identified as a prime environmental factor influencing central body fat mass [10] and with higher markers of inflammation [11], results in line with the present investigation. 
A limitation of the present study is the small number of subjects; however, a strength of the study is its prospective design of the study of the Shuar women in the rainforest. However, we have no similar data from the group that migrated to the Shell Mera community.

\section{Conclusion}

We thus show in this study that the outcome of transition in lifestyle, from collecting wild food products and performing manual agriculture to an urbanized style, is characterized by a deterioration of physical fitness. To reduce this risk, these women need to receive preventive interventions. Findings from a study of supervised exercise training in Amerindian women living in poor urban areas have favorable benefits related to cardiovascular risk factors including improved physical fitness [12]. Thus, exercise may be a low-cost safe therapy to prevent negative effects of market integration.

\section{Acknowledgments}

Florence Judd, Hospital Vozandes del Oriente Shell, Shell Mera, Ecuador, participated in the design and data collection and provided information to the participants.

\section{References}

1. Pavan L, Casigilia E, Braga LM, Winnicki M, Puarto M, et al. (1999) Effects of a traditional lifestyle on the cardiovascular risk profile: the Amondava population of the Brazilian Amazon. Comparison with matched African, Italian and Polish populations. J Hypertens 17:749-756.

2. Tavares EF, Vieira-Filho JP, Andriolo A, Sañudo A, Gimeno SG, et al. (2003) Metabolic profile and cardiovascular risk patterns of an Indian tribe living in the Amazon Region of Brazil. Hum Biol 75: 31-46. [Crossref]
3. Lindgarde F, Widen I, Gebb M, Ahren B (2004) Traditional versus agricultural lifestyle among Shuar women of the Ecuadorian Amazoneffects on leptin levels. Metabolism 53:1355-1353.

4. Pisor AC, Gurven M, Blackwell AD, Kaplan H, Yetish G (2013) Patterns of senescence in human cardiovascular fitness: $\mathrm{VO} 2 \mathrm{max}$ in subsistence and industrialized populations. Am J Hum Biol 25: 756-769. [Crossref]

5. Ahmadi-Abhari S, Luben RN, Warenham NJ, Khaw KT (2013)Seventeen-year risk of all-cause and cause-specific mortality associated with C-reactive protein, fibrinogen and leukocyte count in men and women: the EPIC-Norfolk study. Eur J Epidemiol 28:541-550.

6. LaMonte MJ, Durstine JL, Yanowitz FG, Lim T, DuBose KD, et al. (2002) Cardiorespiratory fitness and C-reactive protein among a tri-ethnic sample of women. Circulation 106: 403-406. [Crossref]

7. Lindgärde F, Vessby B, Ahrén B (2006) Serum cholesteryl fatty acid composition and plasma glucose concentrations in Amerindian women. Am J ClinNutr 84: 1009-1013. [Crossref]

8. Ross EB (1978) Food taboos, diet, and hunting strategy: the adaptation to animals in Amazon cultural ecology. Curr Anthropol 19:1e37.

9. Dufour DL (1991) Diet and nutritional status of Ameridians: a review of the literature. Cad Saude Publica 7: 481-502. [Crossref]

10. Nicklas BJ, You T, Pahor M (2005) Behavioural treatments for chronic systemic inflammation: effects of dietary weight loss and exercise training. CMAJ 172:11991209.

11. Kullo IJ, Khaleghi M, Hensrud DD (2007) Markers of inflammation are inversely associated with VO2 max in asymptomatic men. J Appl Physiol102: 1374-1379. [Crossref]

12. Lindgärde F, Ahrén B (2007) Improved metabolic risk markers following two 6-month physical activity programs among socioeconomic marginalized women of native American Ancestry in Lima, Peru. Diabetes Care30:2230-2232.

Copyright: $(\mathbb{2} 2017$ Lindgärde F. This is an open-access article distributed under the terms of the Creative Commons Attribution License, which permits unrestricted use, distribution, and reproduction in any medium, provided the original author and source are credited. 